\title{
Influence of age of mice on the susceptibility to murine schistosomiasis infection
}

Dorcas S.Yole ${ }^{1}$, Stephen K. Gikuru ${ }^{2}$, Emmanuel O. Wango, ${ }^{3}$ Kiio Kithome ${ }^{1}$, Simon Kiarie ${ }^{1}$ and Moses Limo $^{2}$,

1. Institute of Primate Research (I.P.R.), P.O. Box 24481, Karen, Nairobi, Kenya. 2. Egerton University, Department of Biochemistry \& Molecular Biology, P.O. Box 536 Njoro, Kenya. 3. University of Nairobi, Department of Animal Physiology, P.O. Box 30197, Nairobi, Kenya.

Corresponding Author Dr Dorcas S. Yole,Institute of Primate Research (I.P.R.), P.O Box 24481, Karen, Nairobi, Kenya,Telephone No.: 254020 882571-4;Fax: 254020 882546;E-mail: dsyole@ipr.or.ke

\section{SUMMARY}

Intensity of human schistosomiasis infection increases with age, a peak being attained at early puberty. Hormones could be involved in the age-related changes in susceptibility to schistosomiasis. Male BALB/c mice were infected with Schistosoma mansoni either before or after puberty and worm numbers, cellular immune responses, hormonal levels and pathology analysed. Pre-puberty infected mice had a significantly higher number of adult worms $(p<0.05)$, more severe granulomas, higher mortality rate and higher proliferative responses as compared to postpuberty infected mice. Levels of the hormones were lower in the pre-puberty infected mice as compared to the post-puberty group early in the infection. Plasma levels of testosterone and luteinizing hormones decreased significantly $(p<0.05)$ in infected mice when compared to controls. Susceptibility to $S$. mansoni in male BALB/c mice seems to be influenced by levels of testosterone and leutenizing hormone at infection. Albeit, an infection with $S$. mansoni seems to lower the hormonal levels.

[Afr J Health Sci.2006; 13:47-54]

\section{Introduction}

Epidemiological studies in different geographical regions of the world have demonstrated similarity in age-related susceptibility to schistosome infection in human populations [1]. Data from these communities indicate an increase in intensity of human schistosomiasis that peaks at early puberty. Hormones control many physiological changes during puberty. Gonadotrophins are responsible for the onset of puberty. A rise in adrenal androgens, which are the secondary sex hormones, occurs in both sexes in early puberty [2]. Various investigators have demonstrated changes in levels of some hormones in $S$. mansoni infected humans and experimental animals. Female mice have been found to be more susceptible to $S$. mansoni infection than male mice and this has been attributed to higher levels of testosterone in males [3, 4]. Testosterone was found to be abnormally low in chronically infected hepatosplenic schistosomiasis patients [5]. Dehydroepiandrosterone (DHEA), an adrenal steroid, is associated with puberty in mice and other mammals [2]. Administration of DHEA in mice offered about $50 \%$ protection in mice challenged with $60 \mathrm{~S}$. mansoni cercariae [2]. Serum levels of both DHEAS and testosterone on the day prior to infection negatively 
correlated with schistosome worm burden [2]. Baboons with primary infections (which had higher worm recovery and oviposition rates, and large hepatic egg granulomas) had decreasing levels of DHEA as infection progressed compared with uninfected or re-exposed baboons [6]. When cercariae, schistosomula and adult worms of $S$. mansoni were exposed in vitro to DHEA, cercariae were found to be more susceptible to damage by DHEA than schistosomula or adult worms [7]. Urinary schistosomiasis has been reported to cause delay in puberty in human males [8]. In contrast, mice with $S$. mansoni infection showed increased levels of testosterone [9].

In addition, a hypothalamic neuropetide hormone, somatostatin has been associated with severe morbidity in patients suffering from Schistosoma mansoni infection [10]. In a study conducted in Senegal, physiological levels of somatostatin were found to determine disposition of particular individuals towards severe morbidity as opposed to others [10]. In other studies, this neuropeptide hormone is reported to have demonstrated antifibrotic effects in humans and model animals $[11,12$, 13] and has been proposed to have a possible therapeutic role in treatment of chronic schistosomiasis.

Changes in hormone levels during puberty could modulate responses that indirectly influence schistosome infection such as skin thickness, or directly regulate schistosome infection by modulating the protective immune responses $[14,15]$. This study was designed to examine the influence of age on hormonal, immunological and pathological responses in murine schistosomiasis.

\section{Materials and Methods}

\section{Host and parasite}

$S$. mansoni isolate used in the present study originated from infected Kenyan patients and was routinely maintained in Biomphalaria pfeifferi and baboons at the Institute of Primate Research (IPR), Karen, Nairobi. The cercariae obtained from this parasite isolate were used to initiate infection in BALB/c mice for this study.

\section{Experimental animals and design}

Male BALB/c mice acquired from Institute of Primate Research (I.P.R.) Animal Resources Department were housed in cages, in groups of five per cage. They were maintained on a commercial diet and water ad libitum. The animals were under a daily natural dark/light cycle of approximately $12 \mathrm{~h} / 12 \mathrm{~h}$ at the ambient temperatures of $20 \pm 1{ }^{\circ} \mathrm{C}$, and a relative humidity of $50-60 \%$. Mice aged 24-25 days old (Pre-puberty, PRE) and 9-15 weeks old (postpuberty, PO) were used in the experiment. Mice in each category were further divided into 2 groups of 30 mice each, representing an experimental group infected with $S$. mansoni and uninfected controls. Mice to be infected with $S$. mansoni were anaesthetized with a mixture of xylaxine (Rompun ${ }^{\circledR} 2 \%$ ) and Ketamine (Rotex Medica GMBH Tritau-Germany) at a dose of $0.05 \mathrm{ml}$ per $20 \mathrm{~g}$ body weight and each mouse received approximately 110 cercariae of $S$. mansoni through penetration of intact skin as described by Smithers and Terry, 1965 [12]. Thereafter, every 2 weeks, 5 mice were randomly selected from each of the $S$. mansoni infected (experimental) groups or each uninfected (control) group and blood was drawn by cardiac puncture from anaesthetized mice and plasma prepared for measurement of hormone levels. Spleens from the anaesthetized mice were also removed, placed in labelled petri dishes containing RPMI-1640 medium (GIBCO Technologist Ltd, Parsely, Scotland) supplemented with $1 \%$ Gentamicin, $1 \%$ glutamine $(2 \mathrm{mM})$ and $1 \%$ 2-mercaptoethanol (5x $10^{-5} \mathrm{M}$; Incomplete medium) and used to isolate cells for proliferation assay described below.

\section{Hormonal assays}

Levels of testosterone and leutenizing hormone (LH) from experimental or control mice in the pre-puberty category or post-puberty category mice were measured in blood plasma 2, 4, 6, 8 and 10 weeks post-infection (PI). Plasma testosterone was determined by a competitive radioimmunoassay technique [17]. The WHO reagents used were first validated for use in mice, and $100 \mu \mathrm{l}$ of each plasma sample was 
used in the assay. The concentrations of plasma LH were determined by a bioassay method [17].

\section{Cell Proliferation assays}

A spleen cell suspension was prepared by placing the spleen on a sterile fine metal sieve in a petri dish containing about $2 \mathrm{ml}$ of sterile Incomplete medium, and squashing it using a 10 $\mathrm{ml}$ syringe piston. The cell suspension was dispersed with a sterile Pasteur pipette. The cells were washed twice at $400 \mathrm{~g}$ for $10 \mathrm{~min}$ at room temperature. The cells were then re-suspended in C10 medium (Incomplete medium fortified with $10 \%$ foetal calf serum) and cell density was adjusted to $3 \times 10^{6}$ cells/ ml. Triplicate culture wells were set up for each animal in 96-well microtiter plates at a density of $3 \times 10^{5}$ cells/ 200 $\mu \mathrm{l}$ of medium/ well. Negative control wells contained only $\mathrm{C} 10$ medium and cells, positive controls received $1 \mu \mathrm{g} /$ well of Concanavalin A (Con A), and test wells were stimulated with 10 $\mu \mathrm{g}$ of Soluble Worm Antigen Preparation, SWAP [18]. All the plates were incubated in 5 $\% \mathrm{CO}_{2} /$ air for $72 \mathrm{~h}$ at $37{ }^{\circ} \mathrm{C}$ before being pulsed with $18.5 \mathrm{kBq}$ of $\left[{ }^{3} \mathrm{H}\right]$ thymidine (Specific activity, $185 \mathrm{kBq} / \mathrm{mM}$, Amersham International PLC, Buckingshire, England). Cells were harvested after a further $18 \mathrm{~h}$ of incubation and the incorporated isotope was measured by liquid scintillation counting. Results were expressed as the differential counts per minute $(\Delta \mathrm{cpm})$ of ConA or SWAP stimulated cultures - cpm of negative controls.

\section{Recovery of adult worms from infected mice}

On week 7 PI, 5 mice from the "experimental" and 5 mice from the "controls" groups in both the pre-puberty and post-puberty mice were perfused for recovery of adult schistosome worms using a modified method of Smithers and Terry [16]. The perfusate from each mouse was collected in a glass petri dish then transferred into a urine jar and the worms allowed to settle. Using a process involving sedimentation and removal of the supernatant by suction pressure, the perfusate was then cleaned in 3 washes with phosphate buffered saline (PBS). After the final wash, the small volume of clear liquid remaining at the bottom of the urine jar after removal of most supernatant was transferred into a clean petri dish, and examined under a dissecting microscope. Worms recovered from each mouse were counted and the numbers recorded. Worm maturation was calculated using the following formula:

Worm maturation $=$ Adult worms recovered after perfusion $\mathrm{x} 100$

Penetrating Cercariae

\section{Histopathological examination}

Livers were taken from mice sacrificed at weeks 7, 10 and 11 PI for gross pathology and histopathological examination. Representative biopsy samples of the liver were fixed immediately in $10 \%$ neutral-buffered formalin. The fixed livers were dehydrated using ethyl alcohol, embedded in paraffin wax and cut into 7 $\mu \mathrm{m}$ thick sections. The sections were stained in haematoxylin and Eosin ( $\mathrm{H} \& \mathrm{E})$. Granulomas were located within sections using Ortholux II Leitz Wetzlar Microscope, Germany. The vertical and horizontal diameters of granulomas containing a visible centrally placed schistosome egg were measured using an ocular micrometer. The average horizontal and vertical diameters were taken to be the mean diameter of the granuloma. A total of 20 granulomas were measured for each mouse.

\section{Results}

\section{Hormonal assays}

The $S$. mansoni-infected pre-puberty infected mice (PRE) had much lower plasma testosterone levels during the first six weeks of infection than $S$. mansoni-infected post-puberty mice, (PO; Fig. 1A). On the other hand, the uninfected postpuberty control mice (POC) exhibited significantly higher testosterone levels than the uninfected pre-puberty controls (PREC) at week 2 and $4(p<0.05)$. Overall, the testosterone levels in the $S$. mansoni-infected groups (PRE or PO) were significantly lower than their corresponding controls $(\mathrm{p}<0.05)$ throughout the entire experiment.

In the case of LH levels, the control postpuberty mice (POC) had much higher LH levels during the first 4 weeks of infection than the levels observed for control pre-puberty mice (PREC; Fig. 1B). Overall, LH levels for $S$. 
mansoni-infected mice in both the PRE and PO categories were significantly lower than those of their corresponding controls (PREC and POC $\mathrm{p}<0.05)$.

\section{Proliferative responses}

Proliferative responses of spleen cells stimulated with Con A were high throughout the sampling period, for both the experimental and control groups, showing at least a 10-fold increase compared to spleen cells stimulated with antigens (data not shown). Whereas, the proliferative responses of spleen cells from the two control groups in the pre-puberty (PREC) or post-puberty (POC) mice to SWAP remained low throughout the experiment, the responses of spleen cells from corresponding groups of $S$. mansoni-infected mice (PRE and PO) to the same antigen were significantly higher, $\mathrm{p}<0.05$ (Fig. 2). Interestingly, spleen cells from $S$. mansoni-infected pre-puberty mice (PRE) had significantly higher responses to SWAP at week 4 and $8(\mathrm{p}<0.01 ; \mathrm{p}<0.001$, respectively $)$ than cells from the $S$. mansoni-infected post-puberty mice (PO).

Fig 1: Hormonal levels: (A) Plasma testosterone level \& (B) Plasma leutenizing hormone (LH) level, of blood plasma obtained from male BALB/c mice infected with $S$. mansoni pre-puberty (PRE) or post-puberty (PO) compared with blood plasma levels from corresponding uninfected controls (PREC, POC)

A
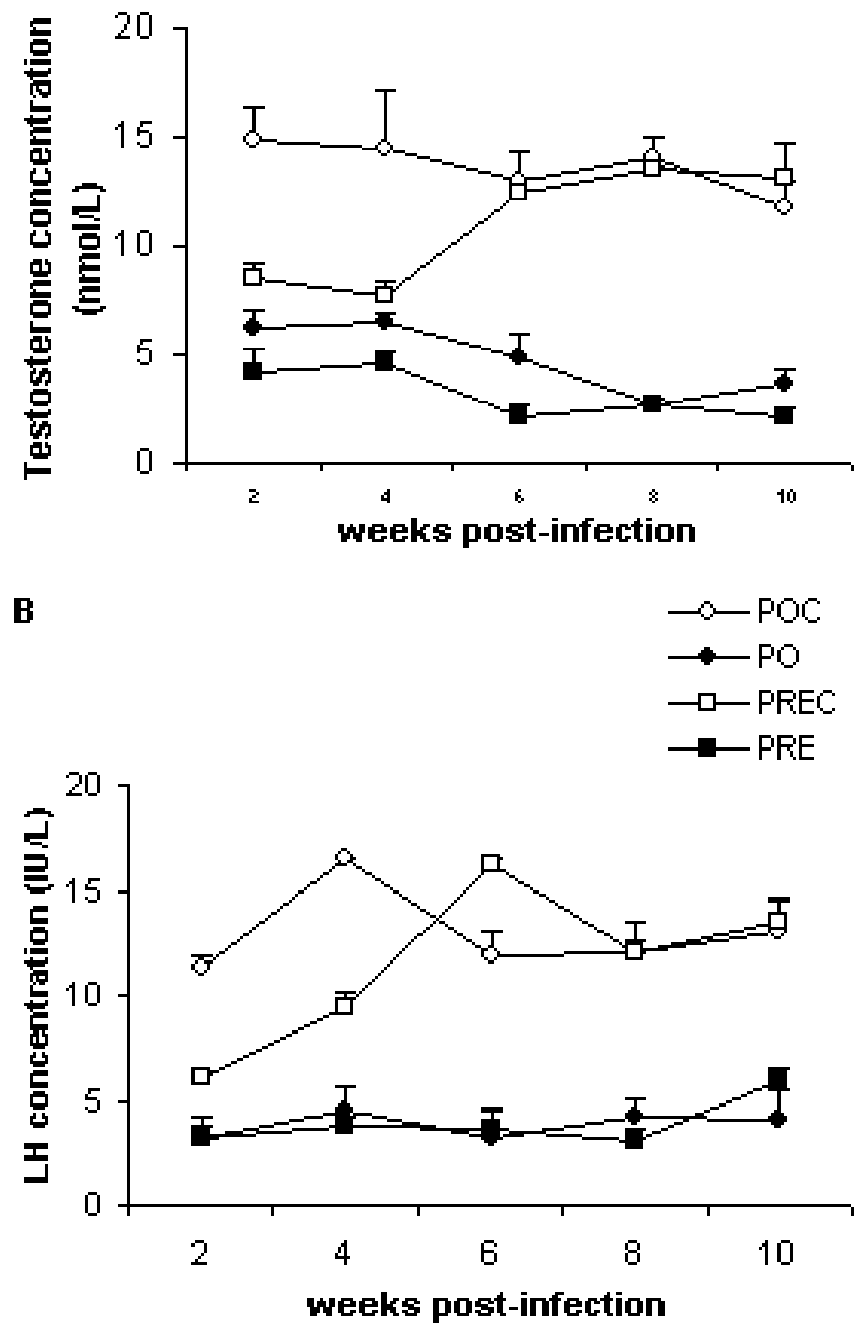
Fig 2: Proliferative responses of soluble worm antigen-stimulated spleen cells from male BALB/c mice infected with $S$. mansoni pre-puberty (PRE) or post-puberty (PO) compared with responses of spleen cells from corresponding uninfected controls (PREC, POC)

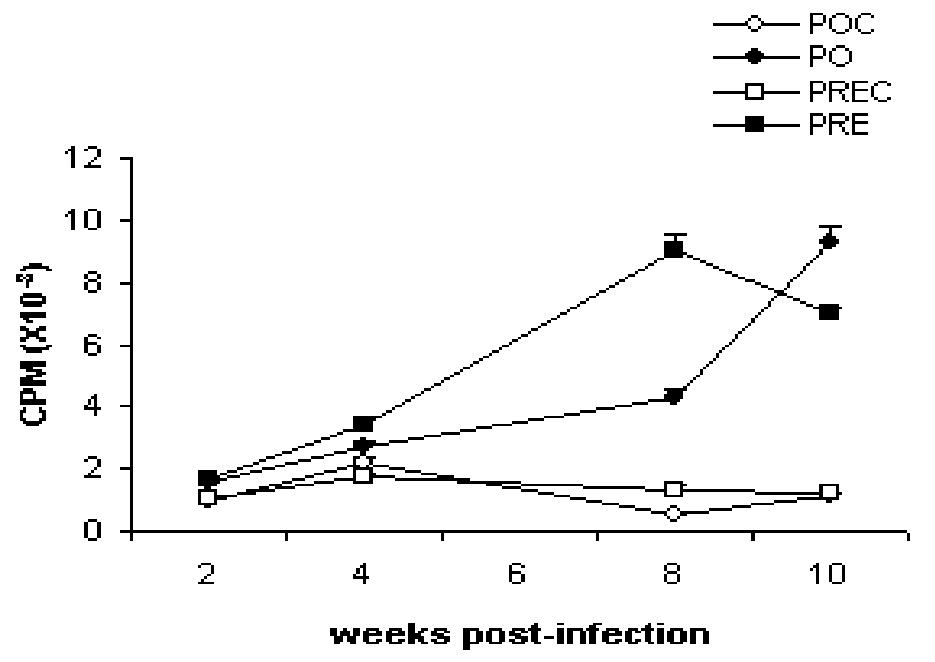

\section{Recovery of adult worms}

The $S$. mansoni-infected pre-puberty mice had a mean worm count of $20 \pm 7$ ( \pm SEM) worms, and this represented a worm maturation of $18 \%$. On the other hand, the $S$. mansoni-infected postpuberty mice had a much lower worm count, with a mean worm count of $10 \pm 2$, and a worm maturation of $9 \%$. A one-way analysis of variance revealed that the number of worms recovered from pre- and post-puberty mice were significantly different $(\mathrm{P}<0.05)$.

\section{Pathology}

The livers of $S$. mansoni-infected animals from both the pre-puberty (PRE) and post-puberty (PO) mice showed severe pathology at weeks 10 and $11 \mathrm{PI}$, but the pre-puberty group had more granulomas. Death as a result of $S$. mansoni infection (as reviewed by post-mortem examination) was only recorded in the infected pre-puberty group (mortality rate of $9.75 \%$ ).

Infected pre- or post-puberty mice attained peak liver granuloma sizes at weeks 10 and 11, respectively (Fig. 3). At all the time points, the granuloma sizes of the pre-puberty mice were much larger than those of the post-puberty mice and by week 7 , the difference was statistically significant $(\mathrm{p}<0.05)$. Pre-puberty mice that died from the infection had significantly larger granuloma sizes $(\mathrm{P}<0.05)$ than the surviving mice from the same group.

\section{Discussion}

Studies of schistosomiasis in endemic areas show that infection rises steadily with age to a peak in the early teenage years, and then falls in adulthood [1]. In the present experiment, prepuberty mice were found to be more susceptible to $S$. mansoni infection than post-puberty mice. Similarly, the level of worm maturation was higher in the pre-puberty mice than in the postpuberty mice. These results are in agreement with those obtained in human studies. The prepuberty group had more granulomas than the post-puberty group. Also, pre-puberty mice had larger granulomas and attained a peak earlier as compared to the post-puberty group. In addition, death due to $S$. mansoni infection was only recorded in the infected pre-puberty group.

The pre-puberty mice that died as a result of $S$. mansoni infection had larger liver granulomas than those in the livers of their living counterparts. A higher worm burden in the pre-puberty group may have led to more granulomas numbers, since more worms would produce more eggs. However, the increase in granuloma size in the pre-puberty group points to involvement of the immune system, since 
granuloma size depends on the number of infiltrating immune cells.

In general spleen cell proliferative responses to SWAP were higher in the $S$ mansoni-infected pre-puberty mice than in the infected postpuberty animals. This could be attributed to the fact that a higher number of worms survived in the pre-puberty animals (thus releasing more antigens) than in the infected post-puberty group. However, other factors that modulate host immune responses such as cytokines could also come into play $[19,20,21,22,23,24]$.

Of relevance is the observation that prepuberty mice had lower levels of testosterone in the early part of infection than the post-puberty mice. This suggests that, decreased levels of testosterone and $\mathrm{LH}$ at the time of infection may be related to increase in worm maturation, cellular responses and increase in numbers and size of granulomas, all reported in the prepuberty infected group.

Fig 3: Granuloma sizes in male BALB/c mice infected with $S$. mansoni pre-puberty (PRE) or post-puberty (PO)

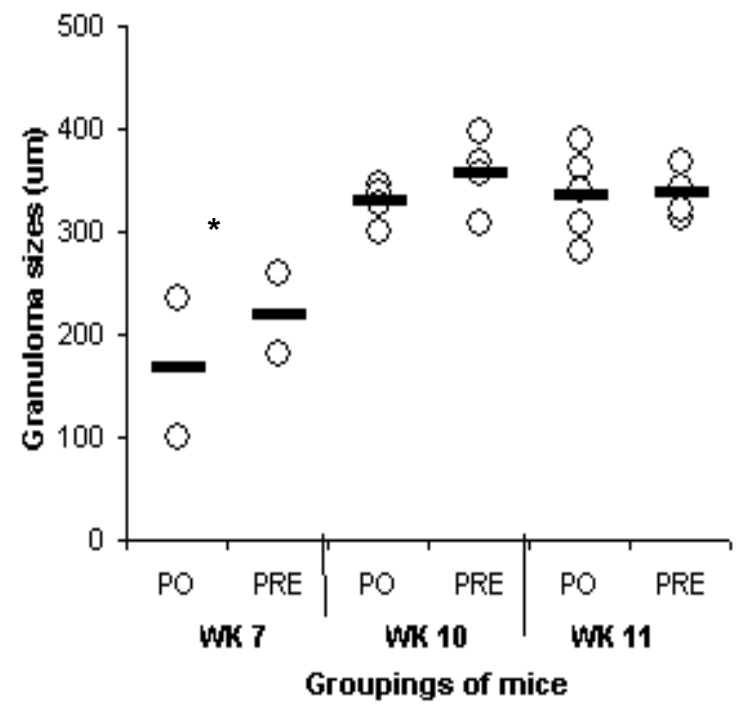

[O: mean granuloma size per mouse; - mean size per group, $\mathrm{n}=5 ; * \mathrm{p}<0.05$ ]

The plasma concentration of both testosterone and luteinizing hormone (LH) decreased significantly in the $S$. mansoni-infected pre- puberty and post-puberty mice compared with the concentrations in their corresponding controls. Possible mechanisms leading to lowered levels of these hormones could be as a result of the central activation of the opiate system by the schistosome infection [25] or production of proopiomelanocortin derived Adreno-cortico-trophic hormone (ACTH) and Bendorphin, by the schistosomes [26]. S. mansoni is capable of producing several proopiomelanocortin (POMC) peptides including beta-endorphin, adrenocorticotropin, melanocyte stimulating hormone, enkephalin and morphine [27]. Some of these opioids are immunosuppressive and may play a role in immune evasion by the parasite and also affect the endocrine profile of the host.

Puberty is associated with rise in gonadal and adrenal steroid hormones. The gonadal steroids such as testosterone influence the immune system [28]. Physiological concentration of testosterone can inhibit the maintenance of proton gradient across the mitochondrial membrane as well as ATP synthesis in schistosomes [29]. Testosterone is reported to be cytotoxic to larvae, but not to adult schistosomes [29]. On the other hand, puberty associated adrenal androgen hormones found in both sexes particularly dehydroepiandrosterone (DHEA) may also influence the immune system [1, 30]. Adrenal androgens have been implicated in a wide range of physiological effects, including regulatory effects on immune system [24] such as enhancing interleukin (IL) -2 synthesis, reversing the depression of interferon gamma [31] and regulating IL- 6 production.

Interactions between the endocrine, nervous and immune system create a network that assists man to fight parasitic diseases. Cytokines, hormones and neuropeptides communicate between the three systems forming this network. A good understanding of hostparasite responses require understanding this network which appear to function via communication and/or reciprocal modulation $[10,11,12]$.

\section{Conclusion}

In conclusion, male BALB/c infected with $S$. 
mansoni before onset of puberty exhibited higher cellular responses, more severe pathology and larger granulomas as compared to the mice infected after the onset of puberty. Mice infected with $S$. mansoni had lower levels of both testosterone and luteinizing hormone, and the decrease was more pronounced in the prepuberty mice. Testosterone could be influencing the mouse immune system and rendering postpuberty mice less susceptible to $S$. mansoni. On the other hand, $S$. mansoni seem to have a mechanism, which lowers the levels of the two hormones in the infected mice.

\section{Acknowledgements}

We would like to thank both Sammy Kisara and Richard Korir for technical assistance and Hezbon Odongo for his input in the hormonal assays.

\section{References}

1. Fulford AJC, Webster M, Ouma JH, Kimani $\mathrm{G}$ and Dunne DW. Puberty and age-related changes in susceptibility to schistosome infection. Parasitology Today. 1998; 14: 2326.

2. Fallon PG, Richardson EJ, Jones FM and Dunne DW. Dehydroepiandrosterone sulfate treatment of mice modulates infection with Schistosoma mansoni. Clinical and Diagnostic Laboratory Immunology. 1998; 5: 251-253.

3. Nakazawa M, Fantappie MR, Freeman GL, Eloi-Sanots S, Olsen NJ, Kovacs WJ, Secor WE and Colley DG. Schistosoma mansoni susceptibility differences between male and female mice can be mediated by testosterone during early infection. Experimental Parasitology. 1997; 85: 233-240.

4. Eloi-Sanots S, Olsen NJ Jr, Correa-Oliveira $\mathrm{R}$, and Colley DG. Schistosoma mansoni: mortality, pathophysiology and susceptibility differences in male and female mice. Experimental Parasitology. 1992; 75: 168-175.

5. Cavaliere H, Leite $Z$ and Medeiros-Neto P. Serum immunoreactive somatomedin-c levels in growth failure and delayed puberty associated with chronic hepatosplenic schistosomiasis. Clinical Endocrinology.
1986; 24: 617-662.

6. Morales-Montor J, Mohamed F, Ghaleb A, Baig S, Hallal-Calleros C and Damian RT. In vitro effects of hypothalamic-pituitaryadrenal axis (HPA) hormones on Schistosoma mansoni. Journal of Parasitology. 2001; 87: 1132-1139.

7. Morales-Montor J, Newhouse E, Mohamed F, Baghdadi A, and Damian RT. Altered levels of hypothalamic-pituitaryadrenocortical axis hormones in baboons and mice during the course of infection with Schistosoma mansoni. Journal of Infectious Diseases. 2001; 183: 313-320.

8. Ibraham II, Barakat RM, Bassiouny HK, Hanna LS, Aboul-Atta AM, Bayad MA, Ghaly IM, Abdalla MI and Hafez ES. Effect of urinary bilharzial infection on male pubertal development and endocrine functions. Archives of Andrology. 1983; 11: 59-64.

9. Abdalah KF, Abdel-Aziz SM, El-Hanshary AS and Afifi LM. Effect of praziquantel on sex hormones levels in murine schistosomiasis mansoni. Journal of Egyptian Society of Parasitology. 1994; 24: 627-632.

10. Stavitsky AB. Regulation of granulomatous inflammation in experimental models of schistosomiasis. Infection and Immunity. 2004; 72:1-12.

11. Chatterjee S and Van Marck E. The role of somatostatin in schistosomiasis: A basis for immmunomodulation in host-parasite interactions. Tropical Medicine and International Health. 2001; 6:578-581.

12. Shyama C, Amodou M and Van Marck. Lower levels of circulating neuropeptide somatostatin in Schistosoma mansoni infected patients may have pathological significance. Tropical Medicine and International Health. 2003; 8:33-36.

13. Chatterjee S, Vrolix GS, Depoortere I, Peeters T and Van Marck E. The therapeutic effect of neuropeptide hormone somatostatin on Schistosoma mansoni caused by liver fibrosis. BMC Infectious Diseases. 2005; 5:45.

14. Ebeling P and Koivisto VA: Physiological importance of dehydroepiandrosterone. 
Lancet. 1994; 343: 1479-1481.

15. Shealy CN: A Review Of Dehydroepiandosterone (DHEA). Integrated Physiological Behavior Sciences. 1995; 30: 308-313.

16. Smithers SR and Terry RJ. The infection of the laboratory hosts with cercariae of Schistosoma mansoni and the recovery of the adult worms. Parasitology. 1965; 55: 695-700.

17. Yole DS, Pemberton R, Reid GD and Wilson RA. Protective immunity to Schistosoma mansoni induced in the olive baboon Papio anubis by irradiated cercariae. Parasitology. 1996; 112: 37-46.

18. WHO. Matched Reagent Programme Method Manual. WHO Special Programme of Research, Development and Training in Human Reproduction. WHO, Geneva, 1996.

19. Grzynch JM, Pearce E, Cheever A, Caulada ZA, Caspar P, Heiny S, Lewis F and Sher A. Egg deposition is the major stimulus for the production of Th2 cytokines in murine schistosomiasis mansoni. Journal of Immunology. 1991; 146: 1322-7

20. Wynn TA, Eltoum I, Cheever AW, Lewis F, Gause W and Sher A. Analysis of cytokine mRNA expression during primary granuloma formation induced by eggs of $S$. mansoni. Journal of Immunology. 1993; 151:1430-1440.

21. Wynn TA, Eltoum I, Oswald IP, Cheever AW and Sher A. Endogenous interleukin 12 (IL-12) regulates granuloma formation induced by egg of Schistosoma mansoni and exogenous IL-12 both inhibits and prophylactically immunizes against egg pathology. Journal of Experimental Medicine. 1994; 179: 1551-1561.

22. Wynn TA, Cheever AW, Jankvic D, Pointdexter RW, Caspar P, Lewis FA and Sher A. An IL-2 based vaccination method for preventing fibrosis induced by schistosome infection. Nature. 1995; 17: 505-511.

23. Oswald IP, Caspar P, Jankovic D, Wynn TA, Pearce EJ and Sher A. IL-12 inhibits Th2 cytokine responses induced by eggs of Schistosoma mansoni. Journal of Immunology. 1994; 153:1707-13.
24. Hoffman KF, Caspar P, Cheever AW, Wynn TA. IFN-gamma, IL-12 and TNF-alpha are required for liver pathology in mice vaccinated with Schistosoma mansoni eggs and IL-12. Journal of Immunology. 1998; 161:4201-10.

25. Kavaliers $M$, Podesta RB, Hirst $M$ and Young B. Evidence for the activation of the endogenous opiate system in hamsters infected with human blood flukes. Life Sciences. 1984; 35:2365-2373.

26. Duvaux-Minet O, Dissons C, Gautron JP, Ptton E, Kordon C and Capron A. The helminth Schistosoma mansoni expresses a peptide similar to human $\beta$ - endorphin and posses a proopiomelanocortin-related gene. The New Biologist. 1990; 2: 93-99.

27. Pryor SC and Elizee R. Evidence of opiates and opiod neuropeptides and their immune effects in parasitic invertebrates representing three different phyla: Schistosoma mansoni, Theromyzon tessulatum, Trichinella spiralis. Acta Biologia Hungaria. 2000; 51: 331-341.

28. Shurrs AHWM and Verheul HAM. Effects of gender on sex steroids on the immune response. Journal of Steroids and Biochemistry. 1990; 35:157-172.

29. Fantappie MR, Galina A, Luis De Mendonca R, Furtado DR, Secor WE, Colley DG, Correa-Oliveira R, Freeman G Jr, Tempone AJ, Lannes De Camargo L and Rumjanek DF. Molecular characterization of a NADH ubiquinone oxidoreductase subunit 5 from Schistosoma mansoni and inhibition of mitochodrial respiratory chain function by testosterone. Molecular and Cellular Biochemistry. 1999; 202:149-158.

30. Besser GM and Thorner MO. Clinical Endocrinology. Mosby-Wolfeg, 1994.

31. Daynes RA, Dudley DJ and Araneo BA. Regulation of murine lymphokine production in vivo II. Dehydroepiandrosterone is a natural enhancer of interleukin 2 synthesis by helper $\mathrm{T}$ cells. European Journal of Immunology. 1990; 20:793-802. 